\title{
DUST IN ACCELERATOR VACUUM SYSTEMS
}

\author{
Darren R.C. Kelly, DESY, Notkestr.85, 22603 Hamburg, Germany
}

\section{Abstract}

Many accelerators of electron beams are susceptible to persistent beam lifetime disruptions, with correspondingly reduced performance. One distinguishes between three prevailing explanations of these disruptions: (1) trapping of positive atomic ions in the negatively charged beam; (2) trapping of small highly positively ionised micro-objects ("dust") in the negatively charged beam; (3) disruptions due to stray magnetic objects trapped in the magnetic field of undulators.

The lifetime disruption of certain electron storage rings that employ ion-getter pump systems are attributed by most researchers to explanation (2), the trapped dust hypothesis. Systematic experimental studies of HERA, PETRA and DORIS reinforce the suspicion that specifically this type of pump system is the culprit.

Examples of beam lifetime disruptions are presented, together with a summary of observations and experiments performed at various afflicted storage rings to investigate dust trapping and the connection between ion getter pumps and dust particle release. Observations of the disrupted beam are found to agree with the dust trapping hypothesis.

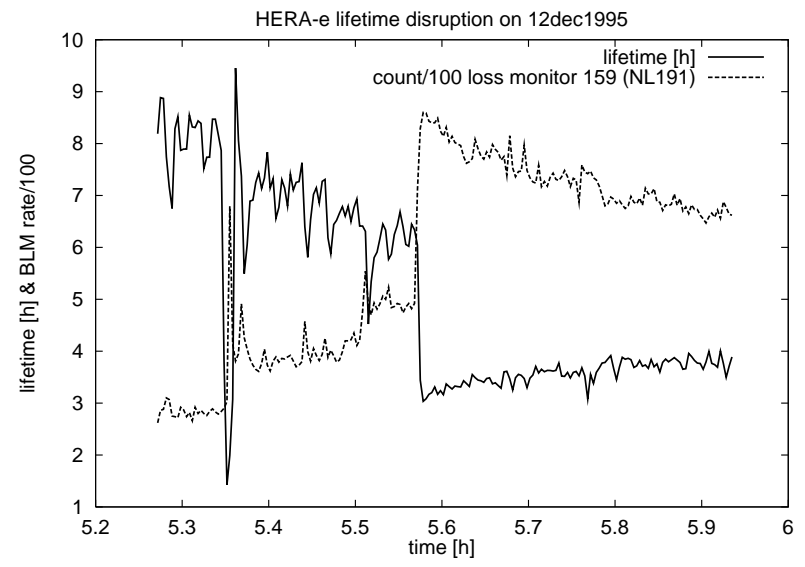

Figure 1: Example of the reaction of a beam loss monitor during a lifetime disruption in HERA-e at injection energy $12 \mathrm{GeV}$ on $12 \mathrm{Dec} 1995$. The beam loss monitor count $R_{i, j}$ divided by 100 for monitor NL191 (number $\mathrm{j}=159$ ) and the lifetime $\tau$ in hours are shown against time $t_{i}$. Reductions in the lifetime coincide with local loss rate increases.

\section{INTRODUCTION}

Various electron storage rings are afflicted by a spurious reduction of the beam lifetime - apparently caused by the deflection of beam electrons by positively charged dust parti- cles of size order $1 \mu \mathrm{m}$ trapped in the electric field potential of the negatively charged beam: Super-ACO [1], TRISTAN AR [2], CESR [3], HERA-e, PETRAII, DORISIII [4, 5], PF [6]. The complementary experience at these machines has built a consistent picture of the symptoms of macroparticle lifetime disruptions, and this picture agrees quantitatively with the dust trapping model detailed in $[3,4]$.

This electron beam lifetime problem is beam charge dependent, i.e. it only occurs with electron beams, as evidenced by the problem-free switch to positron operation in HERA and DORIS.

Observations confirm that the ion getter pumps of all above-mentioned machines are implicated in casting dust particles into the beam pipe. The lifetime disruption can be provoked by switching an ion getter pump on and off, or by abruptly increasing the pump voltage, when a discharge within the pump can be sometimes be observed.

Such lifetime disruptions are not observed in the nonevaporative getter (NEG) pump based storage ring LEP or at ESRF (with NEG pumps and auxilliary lumped ion pumps), with the exception of the possible capture of magnetic dust in undulators [7]. Trials over a limited region of HERA with NEG pumps indicate a strong if not complete reduction of the release of disrupting particles within this NEG pump region.

It will be illustrated in this report that the extensive observations of beam lifetime disruptions in a number of accelerators are well described by the trapped dust hypothesis. However, the formation or liberation of dust particles within the complicated environment of the ion-getter pump chamber is poorly understood. Relatively few direct observations of the processes within these pumps systems are available, whereas many observations of pump configurations and operating conditions conducive to the onset of beam lifetime disruptions have been performed, in particular with HERA, PETRA and DORIS. Such observations are however not sufficient to form a causal model of dust particle release into the beam pipe; our knowledge of the processes on the pump side of the vacuum chamber gas conduction slits is as poor as the available diagnostics. A number of interesting observations and experiments of dust-like disruptions at other afflicted storage rings will also be discussed.

\section{AN EXAMPLE OF LIFETIME DISRUPTIONS IN HERA-E}

Of all available diagnostics of the disrupted beam, the 214 beam loss monitors of HERA [8] are perhaps the most revealing. In Fig. 1 we see an example of a typical life- 


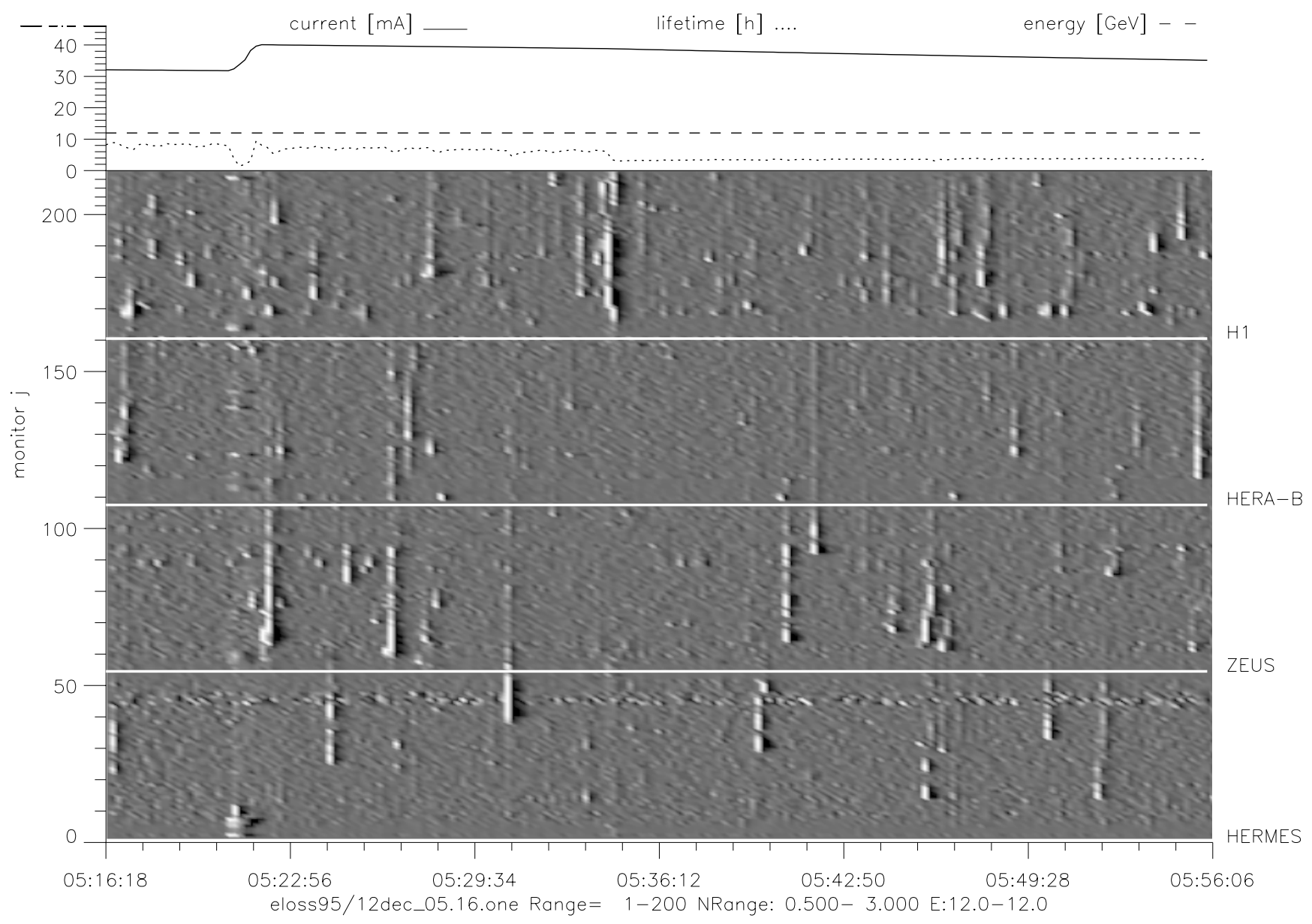

Figure 2: The beam loss monitor count ratio $N_{i, j}=R_{i, j} / R_{i-1, j}$ reveals the influence of dust particles moving longitudinally around the HERA electron ring. The grayscale corresponds to a range $0.5 \ldots 3$ in $N_{i, j}$ at $12 \mathrm{GeV}$, where 1 represents background (no change). The ratios are plotted over the $(i, j)$-plane of BLM monitor numbers $j=1 \ldots 214$ for the entire machine against time $t_{i}$. Current in $\mathrm{mA}$, energy $12 \mathrm{GeV}$ and lifetime in $\mathrm{h}$ are shown on an extended scale

time disruption in HERA-e at injection energy $12 \mathrm{GeV}^{1}$. In this particular HERA-e run a number of abrupt beam lifetime reductions seemed to correlate with increases in the rate $R_{i, j}$ of beam loss monitor number $j=159$ at position NL191 over times $t_{i}$.

More global insight into the dust trappings is obtained when the time development of the beam loss monitor count ratio $N_{i, j}=R_{i, j} / R_{i-1, j}$ of all monitors $j=1 \ldots 214$ around the machine is displayed simultaneously in one diagram for a range of times $t_{i}$. Thus changes in the reaction of each monitor are reflected by departure from $N_{i, j}=1$, which value then represents predominantly the synchrotron radiation background and beam electron losses due to deflection from residual gas molecules in the vacuum cham-

\footnotetext{
${ }^{1}$ In HERA-e at $12 \mathrm{GeV}$ the beam loss monitor reaction to scattered electrons dominates over the loss monitor's reaction to synchrotron radiation, permitting easy recognition of events, whereas at e-p luminosity energy $27.5 \mathrm{GeV}$ transient reactions can be identified but at $3 \%$ of the count are difficult to resolve against the dominant synchrotron radiation background, so examples in this paper are restricted to injection energy $12 \mathrm{GeV}$.
}

ber. In Fig. 2 the count ratio is associated with grey shades for a range $0.5-3$ in $N_{i, j}$ for the same HERA-e run on $12 \mathrm{Dec} 1995$ at $12 \mathrm{GeV}$. The reader will be rewarded by taking a few minutes to peruse and understand this somewhat overwhelming diagram, which illustrates most of the important aspects of the dust trapping problem. A number of features of the disrupting particles can be identified in this diagram:

- The longitudinal flight of many particles can be easily discerned as bright flight trails, and their velocities can be measured to be around 10 to $100 \mathrm{~m} / \mathrm{s}$ (in HERA-e at beam energy $12 \mathrm{GeV}$ and current $30-40 \mathrm{~mA}$ ).

- Both transient and instransient disruptions of the lifetime can be seen to correspond with particles entering the beam. The lifetime is plotted against time on the extended axis.

- It can be seen that there are hundreds to thousands of particles passing through the beam per hour, only a handful of which are permanently trapped. Many 
particles do not survive their flights along an arc, perhaps due to thermal, structural or dynamic instability. There is clearly a narrow stability window.

- The reaction of monitor number 159 (position NL191) shown individually in Fig. 1 can be identified at the instant of lifetime reduction (extended axis).

\section{THE TRAPPED DUST HYPOTHESIS}

Most if not all symptoms of the electron beam lifetime disruption can be quantitatively explained by the prevailing dust trapping model as detailed in [3, 4], which I summarise briefly. Macroparticles, perhaps of $\mathrm{SiO}_{2}$ or metallic oxides from the beam pipe and ion pump surfaces, are cast into the beam pipe by numerous ion pumps at frequent intervals, where they are rapidly ionised and drawn into the electron beam by the beam's strong electric field. The particles are transversely trapped and rapidly reach an equilibrium charge determined by competition between ionisation by beam electrons and deionisation through field evaporation and photoelectron capture. The equilibrium charge obtained by $\mathrm{SiO}_{2}$ particles of sizes $0.1-1 \mu \mathrm{m}$ - as computed by integration of a trapped particle's equation of motion [12] with charge development after [4] - is listed in Table 1 for current $20 \mathrm{~mA}$ and energy $27.5 \mathrm{GeV}$ in HERA-e. The particles oscillate transversely at frequencies of a few $\mathrm{kHz}$. Particles of low mass-to-charge ratio, i.e. of radius $\ll 0.1 \mu$ m are transversely unstable.

The macroparticles are driven downstream by Mœller scattering at about $12 \mathrm{~ms}^{-2}$ [4] until they are possibly trapped in horizontally defocussing quadrupoles by restoring kicks due to the longitudinal asymmetry of the beam bunches from the $\beta$-function gradient there [3]. In HERA particles of radius $<1 \mu \mathrm{m}$ achieve an equilibrium charge meeting this longitudinal trapping criterion.

Table 1: Charge number $Q$, mass number to charge number ratio $Q / A$, and and transverse oscillation frequencies obtained by particles of different radii $R$ trapped in the core of the HERA electron beam at $20 \mathrm{~mA}$ current at $27.5 \mathrm{GeV}$.

\begin{tabular}{lllll}
\hline $\begin{array}{l}R \\
{[\mu \mathrm{m}]}\end{array}$ & $Q$ & $A / Q$ & $\begin{array}{l}f_{x} \\
{[\mathrm{~Hz}]}\end{array}$ & $\begin{array}{l}f_{z} \\
{[\mathrm{~Hz}]}\end{array}$ \\
\hline 1.0 & $5.81 \mathrm{E}+06$ & $1.04 \mathrm{E}+06$ & 1510.88 & 3152.41 \\
0.5 & $4.75 \mathrm{E}+06$ & $1.58 \mathrm{E}+05$ & 3863.99 & 8062.09 \\
0.3 & $4.04 \mathrm{E}+06$ & $4.02 \mathrm{E}+04$ & 7668.93 & 16001 \\
0.1 & $2.72 \mathrm{E}+06$ & $2.23 \mathrm{E}+03$ & 32576.5 & 67969.8 \\
\hline
\end{tabular}

Particles of size $0.1-1 \mu \mathrm{m}$ should be thermally stable in HERA-e at typical current $40 \mathrm{~mA}$ and energy $27.5 \mathrm{GeV}$, although [4] has predicted that these particle might be thermally unstable at very high electron currents $\gg 40 \mathrm{~mA}$.

In view of this model the myriad of activity in the beam loss monitor diagram Fig. 2 becomes understandable. A large number of dust particles with a distribution of sizes are cast into the beam pipe at many locations in the machine. Only the handfull of particles per hour meeting the transverse, longitudinal and thermal stability conditions survive to cause intransient lifetime disruptions, the others leaving merely brief trails of losses as they are swept downstream before melting, falling out of the beam, or becoming structurally unstable.

\section{EXPERIMENTAL INVESTIGATIONS}

A number of chance observations and machine studies at various institutes have added greatly to our knowledge of the lifetime disruption problem, some of which are now briefly described.

Since the inception of CESR sudden lifetime drops have been observed and by comparison of the magnitude of the observed lifetime drops with a model of a trapped macroparticle's equilibrium charge as a function of particle mass the typical particle size was estimated to be of order $1 \mu \mathrm{m}[3]$.

At TRISTAN AR dust particles were dropped into the beam pipe via a $1 \mathrm{~mm}$ nozzle to see whether macroparticles could indeed be trapped in an electron beam [14]. Metal particles consisting of $\mathrm{Cu}, \mathrm{Al}, \mathrm{Ti}$ (sizes $0.1-8 \mu \mathrm{m}$ ) were not trapped, whereas metallic oxides such as $\mathrm{Cu} 0(0.35 \mu \mathrm{m})$, $\mathrm{TiO}_{2}(0.3 \mu \mathrm{m})$, and the NEG compound $\mathrm{Zr}-\mathrm{V}-\mathrm{Fe}(\sim 1 \mu \mathrm{m})$ were trapped for many minutes at currents order $10 \mathrm{~mA}$ and energy $6.5 \mathrm{GeV}$. Particles of diameter $0.5 \mu \mathrm{m}$ consisting of $\mathrm{C}$ (diamond), $\mathrm{SiC}$ and $\mathrm{Al}_{2} \mathrm{O}_{3}$ were trapped even at very low currents (less than $0.1 \mathrm{~mA}$ ).

Surprisingly, the TRISTAN AR beam lifetime was found to be poor after dumping and refilling, and bremsstrahlung signals and electron losses typical of trapped particles were still observed. This "hysteresis" effect has also been observed at HERA, and is not yet understood. Simple considerations predict that the image charge force on a dust particle exceeds the beam electric field force within a few $\mathrm{mm}$ of the vacuum chamber wall for both HERA and TRISTAN AR [3, 15].

During dust trapping investigations at TRISTAN AR bremsstrahlung observations with $\gamma$-ray detectors [15] suggested both longitudinal motion and transverse oscillations of dust particles.

At CERN a troublesome ion pump installed above an electrostatic separator was reported [7]. The pump was found to arc frequently. When the pump was moved below the separator and around a $90^{\circ}$ elbow the problem disappeared.

At Super-ACO a CCD camera $10 \mathrm{~m}$ from an ion pump in a beam-line from Super-ACO was found to be "sandblasted" with titanium and stainless steel after an accidental gas inlet [7].

The degree and frequency of beam lifetime disruptions seems to increase with higher beam energy and beam current. This correlation has been systematically studied in HERA-e [9]. Analysis of the correlation reveals that dozens of particles of radius $0.3 \mu \mathrm{m}$ are typically trapped 
in HERA at current $20 \mathrm{~mA}$ and energy $27.5 \mathrm{GeV}$ resulting in a total lifetime reduction from $10 \mathrm{~h}$ to about $3 \mathrm{~h}$.

It has been suggested [16] that the likelihood of spontaneous ion pump discharge and consequent macroparticle release is related to the number of photoelectrons released per metre from the vacuum chamber surface, a quantity approximately proportional to the product $E \times I$ where $E$ and $I$ are the beam energy and current respectively. This defines a locus for onset of the disruptions, although clearly one is dealing with a stochastic phenomenon not a sharp threshold. There were no indications of lifetime disruptions due to macroparticles at the NEG-pump based LEP storage ring with beam current $8 \mathrm{~mA}$ at $45 \mathrm{GeV}$ [19], whereas lifetime disruptions are prevalent at HERA at current $30 \mathrm{~mA}$ at $12 \mathrm{GeV}$ and at current $13 \mathrm{~mA}$ at $27.5 \mathrm{GeV}$.

Investigations in PETRA showed that a range of adapted ion pumps were capable of causing lifetime disruptions [17], either spontaneously or by abrupt switching of the pump high-voltage. These pump variations included: (1) a regular PETRA ion pump where many cylindrical Penning cells share a common cathode; (2) a regular HERA ion pump where perforated anode foils offer an open discharge region to the side; (3) a HERA ion pump with a baffle between the gas conduction slits and the pump anode, blocking the direct route to the beam pipe; (4) an "inverted" pump with high negative voltage attached to the cathode instead of the anode. A "dummy" pump with a closed, inactive rectangular tube replacing the cylindrical Penning cells did not give rise to lifetime disruptions.

A possible mechanism for dust particle release from ion pumps is the liberation of pump material such as titanium by strong discharges known to occur spontaneously within the pump cells, and provokable by abrupt switching of the pump high voltage. Resistors of strength $100 \mathrm{M} \Omega$ were installed before integrated dipole pumps in HERA in the hope of dissipating the energy of the discharge sufficiently to prevent liberation of particles [18]. However beam lifetime disruptions where found nevertheless to occur frequently and analysis of beam loss monitor responses did not show a reduction in the frequency of particle release into the beam pipe.

It has been shown that both strong repeated beam kicks and a carefully tuned beam excitation sweep with a feedback kicker can restore the disrupted beam lifetime of PETRA and HERA [17, 11, 12]. In HERA, where the lifetime is "multiply-disrupted", presumably by dozens of disrupting particles, the lifetime and the rates in particular beam loss monitors and experiment detectors could be seen to improve in discrete steps as the parameters of the beam excitation were scanned, providing strong support for the dust trapping model. Bremsstrahlung detector observations at PETRA likewise indicated discrete beam disruption.

A clearing field constructed using beam position monitors button electrodes immediately downstream from HERA's horizontally defocussing quadrupoles was shown to deflect the longitudinal flights of particles travelling sufficiently slowly past the clearing field [13]. The location of the BPMs is not suitable for construction of a clearing field capable of improving the beam lifetime in HERA by removing particles trapped within horizontally defocussing quadrupoles, but the experiment provides further support for the dust trapping model. The installation of general clearing-field electrodes to improve the HERA-e beam lifetime is a technically cumbersome option.

\section{CONCLUSION}

A wealth of experimental and observational information has been gathered by researchers at many electron storage rings afflicted by spontaneous beam lifetime disruptions, most of which is consistent with the model of macroparticles (dust) of size order $1 \mu \mathrm{m}$ being trapped in the electron beam.

Yet despite this consistent picture of the symptoms, the cause is not fully understood. As researchers of viral infections know, the tiniest creatures can be responsible for the greatest grievances and warrant the most extensive combat. An elegant, inexpensive solution to the electron beam lifetime problem compatible with general machine operation in HERA is not yet available. Trials indicate that replacing the ion getter pump system with NEG pumps will likely restore good electron beam lifetime. But a deeper understanding of dust generation/liberation in accelerator pump systems would be welcome now that so much is known about dust in electron accelerator beam pipes.

\section{ACKNOWLEDGEMENTS}

Thanks to DESY colleagues and other electron storage ring colleagues too numerous to list for discussions and communications.

\section{REFERENCES}

[1] P. Marin. 'Microlosses of beam current on Super-ACO operated with electrons', LURE, ORSAY RT/90-01 (1990)

[2] H. Saeki, T. Momose, and H. Ishimaru. 'Observations of dust trapping phenomena in the TRISTAN accumulation ring and study of dust removal in a beam chamber', Rev. Sci. Instrum. 62, 874 (1991)

[3] D. Sagan. 'Mass and charge measurement of trapped dust in the CESR storage ring', Nucl. Instrum. Methods. A330 371 (1993)

[4] F. Zimmerman. 'Trapped dust in HERA and prospects for PEP-II', Technical Report PEP-II AP Note No.: 8-94, (Stanford Linear Accelerator Center, 1994).

[5] D. R. C. Kelly. 'Characterisation of lepton beam lifetime behaviour in HERA', Technical Report DESY HERA 95-01', (Deutsches Elektronen Synchrotron, 1995).

[6] K. Fuke 'Photon Factory activity report \#6',R-1, 1988

[7] P. Marin. 'Problems encountered with stray micro-objects in various ultra-high vacuum systems', LURE, ORSAY RT/9304 (1993) 
[8] Bialowons, W., Ridoutt, F., and Wittenburg, K. (1994), 'Electron beam loss monitors for HERA', in Fourth European Particle Accelerator Conference (EPAC94), Vol. 2, p. 1628, World Scientific

[9] D. R. C. Kelly. 'Many-event lifetime disruption in HERA and DORIS', Technical Report DESY HERA 95-02, (Deutsches Elektronen Synchrotron, 1995).

[10] D. Sagan. 'Some aspects of longitudinal motion of ions in electron storage rings', Nucl.Instrum.Meth.A307 171 (1991)

[11] D. R. C. Kelly. 'The effect of beam excitation on the HERA electron beam lifetime disruption'. In Fifth European Particle Accelerator Conference (EPAC96).

[12] D. R. C. Kelly. 'The effect of transverse beam excitation and kicking on the HERA electron beam lifetime disruption'. To be submitted to Nucl. Instrum. Methods.A (1997)

[13] D. R. C. Kelly. 'The effect of a static clearing field on trapped dust particles in the HERA electron ring', To be submitted to Nucl. Instrum. Methods.A (1997)

[14] K. Kanazawa, (TRISTAN) pers.communication, (1995)

[15] H. Saeki, T. Momose, and H. Ishimaru. 'Motions of trapped dust particles around the electron beam in the TRISTAN accumulation ring', Rev. Sci. Instrum. 62, 11 (1991)

[16] J. Kouptsidis, 'Lebensdauerprobleme in Elektronenspeicherringen', in HERA Seminar, Bad Lauterberg DESY (1995)

[17] H. Ehrlichmann, 'Untersuchung zur Elektronenlebensdauer in PETRA', in HERA Seminar, St.Englmar DESY (1996)

[18] W. Bialowons, 'HERA Betrieb mit Elektronen', in HERA Seminar, Hamburg DESY (1997)

[19] CERN, LEP machine study 10 June 1995: S. Myers, F. Willeke, J. Kouptsidis, W. Bialowons, H. Ehrlichmann, K. Wittenburg, D. Kelly. 